\title{
The 'fat' C2 sign
}

Amarjit Kaur Bajwa, MB BS, FCRad (Diag) SA

Anthony Briede, MB BCh, FCRad (Diag) SA

Department of Diagnostic Radiology, Chris Hani Baragwanath Hospital and University of the Witwatersrand, Johannesburg

The 'fat' $\mathrm{C} 2$ refers to the apparent increase in the distance between the anterior and the posterior margins of the $\mathrm{C} 2$ vertebra when compared with the similar two margins of the $\mathrm{C} 3$ vertebrae (Fig. 1) on a lateral cervical spine X-ray in a trauma setting. ${ }^{1}$ This is caused by an obliquely

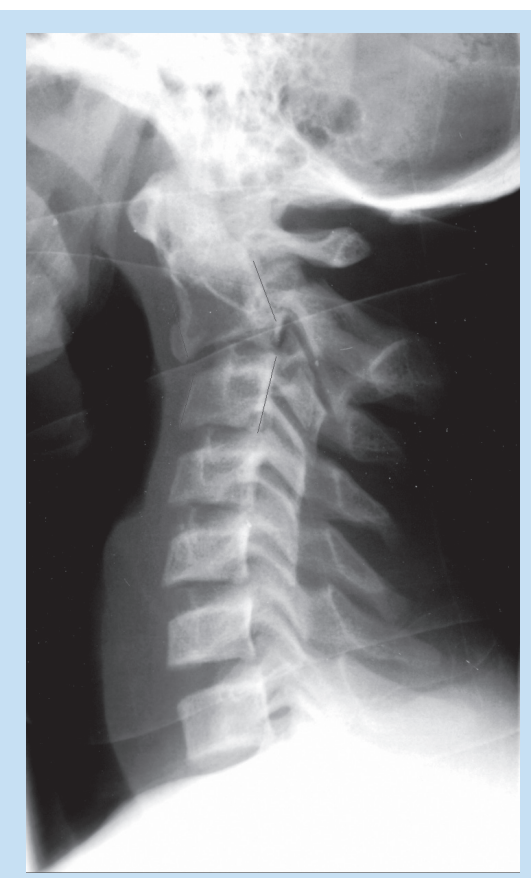

Fig. 1. The lateral cervical spine radiograph shows that the anteroposterior diameter measured between anterior and posterior margins of C2 is more than the same distance at C3 (there is no significant soft-tissue swelling present).

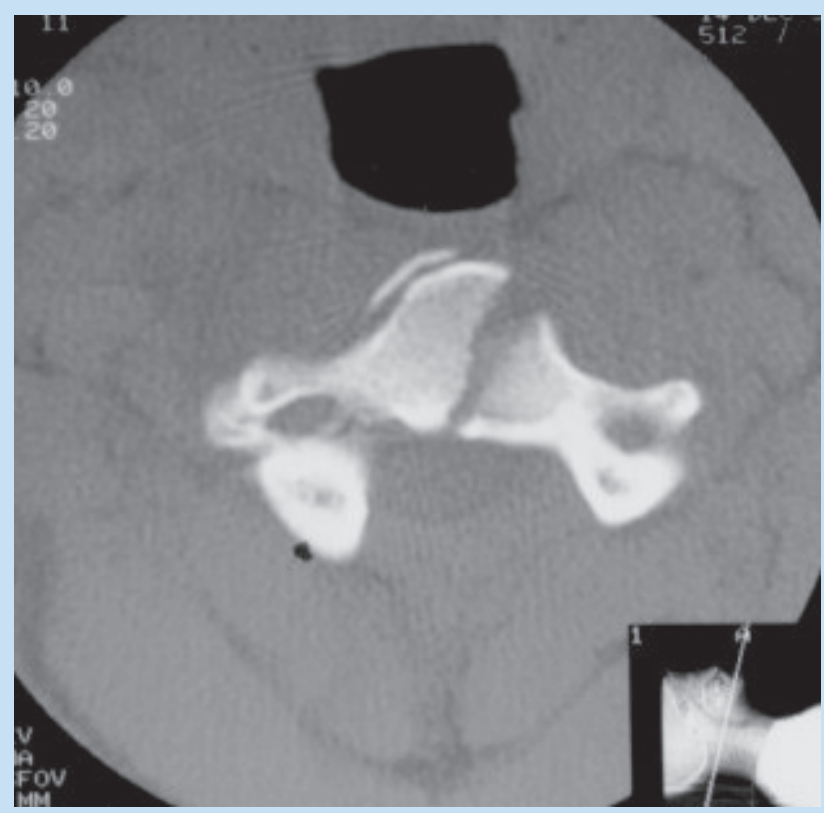

Fig. 2. Axial computed tomographic (CT) scan of the same patient shows the oblique fracture not well appreciated on lateral film.

oriented fracture which may be obscured on anteroposterior or lateral film as the fracture lines are not perpendicular to the plane of the radiograph (Fig. 2).

The applied traumatic forces may cause the interruption of the anterior (with primarily hyperflexion injury) and posterior (with primarily hyperextension injury) or both (combined injury) margins with apparent enlargement of the $\mathrm{C} 2{ }^{2}$

The recognition of fat $\mathrm{C} 2$ sign is important as these fractures are unstable with ligamentous disruption. ${ }^{2}$ Delay in diagnosis may be devastating to the patient.

1. Smoker WRK, Dolan KD. The 'fat' C2: a sign of fracture. AJR 1987; 148: 609-614.

2. Harris JH, Mirvis SE. Injuries of diverse or poorly understood mechanisms. In: The Radiology of Acute Cervical Spine Trauma. 3rd ed. Baltimore, Md: Williams \& Wilkins, 1996; 421-472.
Amarjit Kaur Bajwa, MB BS, FCRad (Diag) SA Department of Diagnostic Radiology, Chris Hani Baragwanath Hospital and University of the Witwatersrand, Johannesburg

Flowing blood causes a ghosting artifact in the phase-encoding direction. Gradient recalled echo (GRE) sequences are much more susceptible to flow artifacts than spin echo (SE) sequences are. ${ }^{1}$ The use of saturation bands can decrease these artifacts. These can also be minimised using flow compensation or gradient moment nulling. ${ }^{1}$ However, this artifact can be used to diagnostic advantage.

\section{The pulsation sign}

Pseudoaneurysm or avascular lesion can be missed on magnetic resonance (MR) imaging even with IV contrast. Surrounding haematoma of different stages can complicate the matter even further (Fig. 1a). However the recognition of ghosting artifact in the phase-encoding direction in line with the lesion along with signal void on the standard SE sequences are important clues to the diagnosis (Figs $1 \mathrm{~b}$ and 2).

Thus pulsation artifact can be used as a diagnostic clue, especially in musculoskeletal imaging where it is normally not that marked.

1. Zhuo J, Gullapalli Rao P. MR artifacts, safety and quality control. Radiographics 2006; 26: 275-297. 


\section{TODAY'S TWO SIGNS}
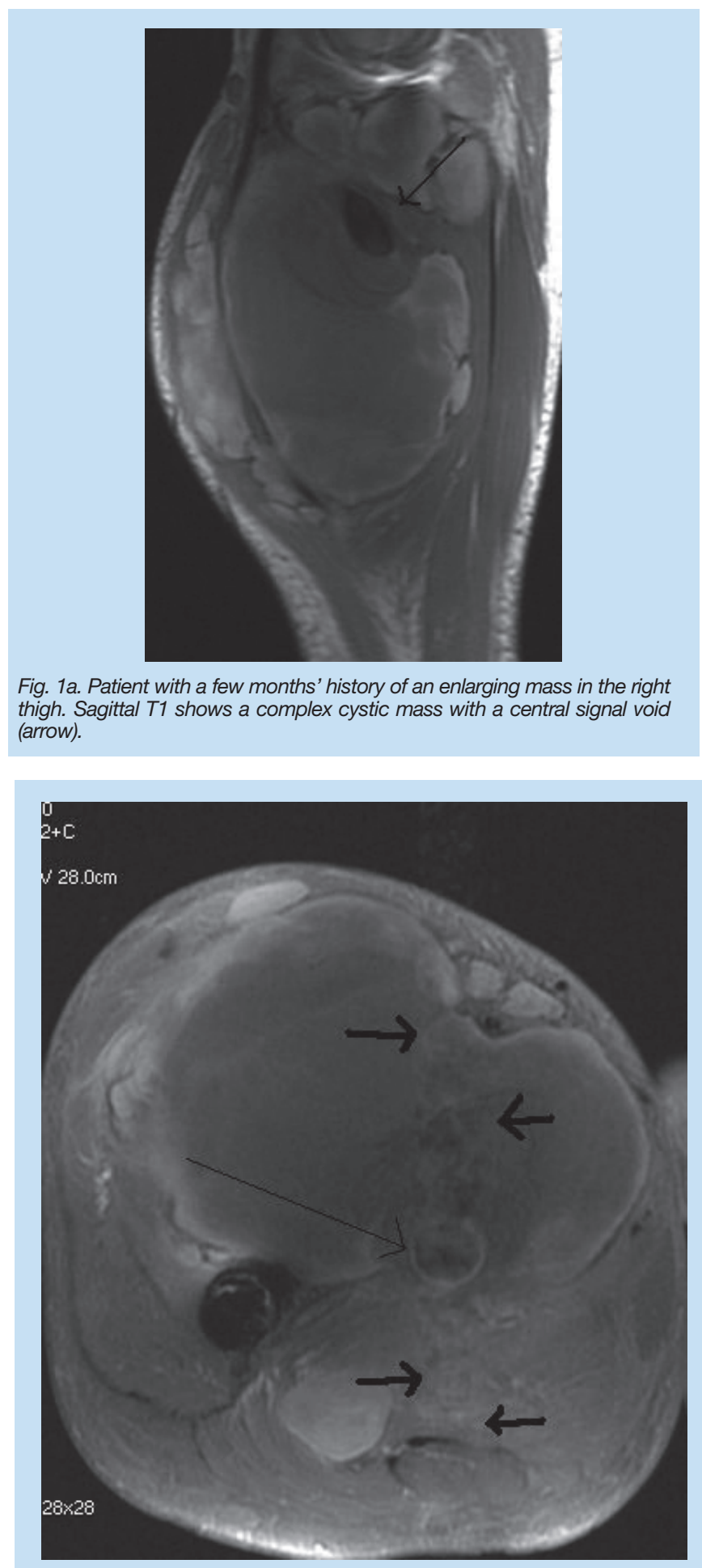

Fig. $1 b$. Post contrast axial T1 shows central minimal enhancement (arrow) and the pulsation artifact in the phase-encoding direction (AP) indicating the vascular origin of the lesion (thick arrows).

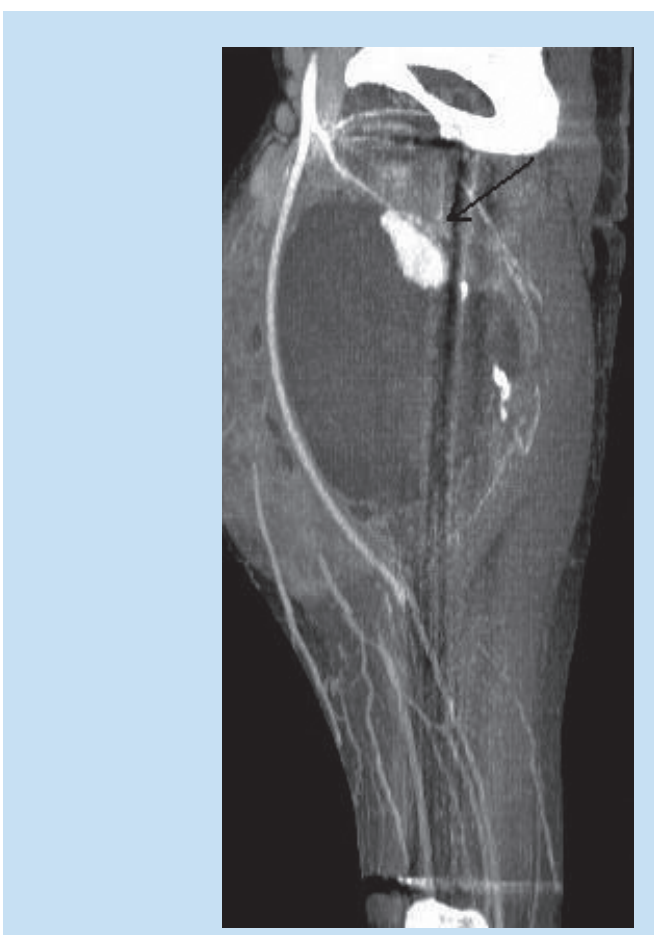

Fig. 2. CT angiography with sagittal multiplanar reconstruction confirms a pseudoaneurysm of the profunda artery (arrow). 